\title{
Novel therapeutic approaches based on lactaptin action
}

\author{
O. Koval ${ }^{1,2 *}$, O. Volkova ${ }^{3}$, G. Kochneva 4 , S. Kulemzin 3 , A. Gorchakov ${ }^{1}$,

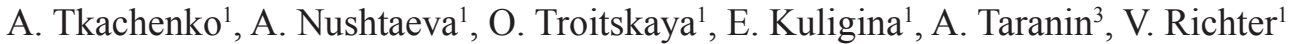 \\ ${ }^{1}$ Institute of Chemical Biology and Fundamental Medicine SB RAS, Novosibirsk, Russia \\ ${ }^{2}$ Novosibirsk State University, Novosibirsk, Russia \\ ${ }^{3}$ Institute of Molecular and Cellular Biology SB RAS, Novosibirsk, Russia \\ ${ }^{4}$ State Research Center of Virology and Biotechnology "Vector" Rospotrebnadzor, Koltsovo, Russia \\ *e-mail:o.koval@niboch.nsc.ru
}

Key words: apoptosis, lactaptin, oncolytic viruses, immunotherapy

Motivation and Aim: Immunotherapeutic approaches become a new hope for cancer treatment. Clinical success was demonstrated for adoptive cell transfer of $\mathrm{T}$ cells expressing chimeric antigen receptors (CARs) as well as for oncolytic viruses. However, both technologies are needed to be improved. Lactaptin was discovered as a molecule specifically inducing death of various cancer cells in vitro and in vivo. So we propose to use lactaptin transgene for armoring CAR NK-cells and vaccinia virus.

Methods and Algorithms: We have engineered double recombinant vaccinia virus (VV) coding human granulocyte-macrophage colony-stimulating factor (GM-CSF) and apoptosis-inducing protein lactaptin (VV-GMCSF-Lact). To engineere "armored" CAR NK-cells secreting an anticancer peptide lactaptin at the first stage we designed lentiviral constructs allowing stable transduction of human cell lines with cassettes encoding two secreted forms of lactaptin.

Results: VV-GMCSF-Lact activated a set of critical apoptosis markers in infected cells: phosphatidylserine externalisation, caspase $-3,-7$ activation, DNA fragmentation, up-regulation of pro-apoptotic protein BAX and efficiently decreased mitochondrial membrane potential of infected cancer cell. Investigating immunogenic cell death (ICD) markers in cancer cell infected with VV-GMCSF-Lact we demonstrated VV was efficient in calreticulin and HSP70 protein externalisation, cellular high-mobility group box-1 (HMGB1) decreasing and ATP secretion. The analysis of antitumor activity against advanced MDA-MB-231 tumor in mice revealed that VV-GMCSF-Lact delay tumor growth up to $94 \%$.

Lactaptin was successfully produced in HEK293T and YT cell lines. Its in vitro activity in the conditioned media was measured against a panel of sensitive cancer cells: MDAMB-231 breast adenocarcinoma, PC3 prostate cancer and T98G glioblastoma. We evaluated that lactaptin from conditioned media showed greater than 50 -fold increase in cytotoxicity compared to the recombinant lactaptin produced in E. coli.

Conclusion: We demonstrated that lactaptin has a great potential for improving immunotherapeutic approaches against cancer.

Acknowledgements: Supported by the VolkswagenStiftung Grant No. 90315, by the Russian Ministry of Education and Science, Agreement 14.604.21.0169 (unique project identifier RFMEFI60417X0169). 